\title{
Mobile Sink and Ant Colony Optimization based Energy Efficient Routing Algorithm
}

\author{
Navpreet Kaur \\ Student of M.Tech C.S.E \\ H.no 194 Akash Avenue Amritsar
}

\author{
Jatinder Pal Sharma \\ Asstt. Prof C.S.E \\ G.I.M.E.T \\ Amritsar
}

\begin{abstract}
In this paper, an Ant colony optimization based energy efficient routing algorithm has been proposed. Principle improvement has been done for energy efficient routing algorithm. Additionally the use of the compressive sensing also increases the performance further. The compressive sensing uses data fusion to remove redundant data from sensor nodes. So it improves the results further. In the end to evaluate the effectiveness of the proposed technique further the effect of the scalability of number of nodes has also been considered.
\end{abstract}

\section{Keywords}

WSN-wireless sensor network, ACO-ant colony optimization, ERA-energy aware routing algorithm.

\section{INTRODUCTION}

WSN means for Wireless sensor network .WSN consists of a large number of nodes which communicates together through sensing and by monitoring the physical world. An instant sensor network is composed of a massive no. of nodes which are employed for sensing the physical world. The different sensor nodes are arranged in such a way so that they'll sense and monitor the movement in the network. In WSN, sensor nodes collects the info and send the collected data to the key node which will be called as sink and it has the maximum energy and then sink node collects the info which will be sent by the sensor nodes. These networks are mobile ad hoc networks and theses networks also don't take the burden of sender and receiver. These networks, thus advances the time of a wireless network. It generates force balancing among the whole network. Wireless sensor networks are utilized in a numerous applications like medical, military.

\section{CLUSTERING}

In clustering various sensor nodes are grouped in the form of clusters. In each and every cluster there is a cluster head which acts as a leader of the cluster. All nodes which belongs to that particular cluster have to send their respective data to the cluster head of that particular cluster and then cluster head is responsible for aggregating the data and sends that aggregated data to base station directly. Only cluster heads have the ability to perform direct communication with base station. Clustering is useful for Useful energy consumption and it also declines the communication overhead for both single and multi-hop networks.

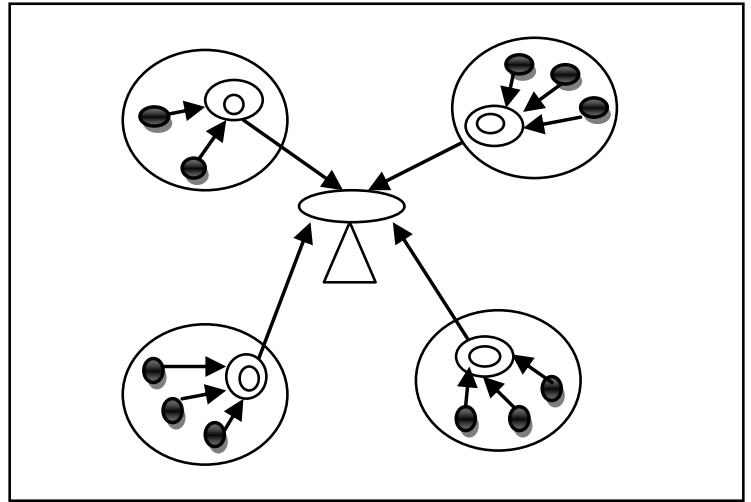

Fig 1: Clustering architecture ( [1])

\section{VARIOUS CLUSTERING PROTOCOLS}

LEACH [9] is just a distributed algorithm for clustering. In this technique, various sensor nodes send their data for their respective cluster heads, and then your cluster heads aggregates the data and then compress the information and delivers the aggregated data to the beds base station. Only cluster head is accountable for directly communicating with base station. In this protocol, cluster head selection is mainly based mostly on the exact distance of the node and the cluster head. The node which can be closest to base station is selected because the "cluster head $\&$ rd quo .All nodes that are cluster members can communicate with the cluster head following TDMA strategy, in accordance to the schedule which can be created by the cluster head. They did by using the minimum energy which must reach the cluster head. Each node independently has the proper to take decision what node is usually to be selected as "cluster head" for a specific cluster. This protocol is useful for reducing the overhead and also used to minimize the energy.

\section{EECS (Energy Efficient Clustering Scheme):}

EECS [6] technique is same to that particular of LEACH-like clustering scheme, in this the network is separated into various group of clusters and there's one cluster head in each and every cluster/. Communication that takes between the cluster head and base station is direct. In this nodes are accountable for broadcasting the messages for their respective neighboring nodes and the node which has maximum residual energy is selected as a bunch head. This protocol enhances the time of the network and also enhances the distribution of energy on the list of network. 


\section{DEEC (Distributed Energy Efficient Clustering):}

In DEEC [6], the cluster-heads are elected on the basis of probability based on the ratio between residual energy of each node and the typical energy of the complete network. The nodes which have high initial and residual energy will have more chances to be selected as cluster-heads compared to the nodes with low energy.

\section{ANT COLONY OPTIMIZATION}

Ant colony optimization is a Meta-heuristic technique of finding the best optimal solution in accordance with the natural actions of ants including their mechanism of collaboration and variation. At first, the ants wander randomly or arbitrarily. When an ant finds a source of meal, it walks back to the colony leaving "markers" (pheromones) that show the path has meal. When other ants come across the markers, they are like to adhere the same direction with a certain probability. If they do, they then fill the same direction with their own markers as they bring the food back. As more ants find the path, it gets more powerful until there are a couple streams of ants visiting various meal sources near the colony. The ACO algorithm is based on following three things.

i.Each direction is followed by the ant is associated to the candidate solution.

ii. The amount of pheromone deposited is proportional to the quality of corresponding candidate solution of the target problem.

iii. When there are more than one path, the ant selects the direction with more pheromones placed on it.

\section{LITERATURE SURVEY}

$\mathrm{Li}$, Shu-song Dong, and Xiang-ming Wen [1] proposed an energy efficient clustering routing (EECR) algorithm for wireless sensor network. The algorithm can divide a sensor network into a few clusters and select a cluster head base on weight value that leads to more uniform energy dissipation evenly among all sensor nodes. Simulations and results show that the algorithm can save overall energy consumption and extend the lifetime of the wireless sensor network. Jin, Yan et al. [2] studied the connected, coverage problem given a specific network coverage ratio under border effects. They considered the scenario where the sensor nodes are distributed in a circle-shaped region uniformly. They first derived the network coverage provided by $N$ sensor nodes by the mathematical formulae exactly. The lower bound of the network connectivity probability is also derived. Since sensor nodes are equipped with energy-limited batteries, energy conservation in such networks is of paramount importance to prolong the network lifetime. Accordingly, we then propose a location-independent, energy-efficient routing algorithm ECCRA which achieves the required network coverage and sensor connectivity simultaneously. The extensive simulation results demonstrate that our algorithm is correct and effective. Jang, Yong-Jae et al. [3] proposed an energy efficient routing algorithm that establishes routing table using broadcasting which is cyclically transmitting and conduct performance analysis with simulation method. Zeydan, Engi et al. [4] investigated the reduction in the total energy consumption of wireless sensor networks using multi-hop data aggregation by constructing energy-efficient data aggregation trees. We propose an adaptive and distributed routing algorithm for correlated data gathering and exploit the data correlation between nodes using a game theoretic framework. Routes are chosen to minimize the total energy expended by the network using best response dynamics to local data. The cost function that is used for the proposed routing algorithm takes into account energy, interference and in-network data aggregation. The iterative algorithm is shown to converge in a finite number of steps. Simulations results show that multi-hop data aggregation can significantly reduce the total energy consumption in the network. Zungeru, Adamu Murtala et al. [5] presents a comprehensive survey and comparison of routing protocols in WSNs. The first part of the paper surveys state-of-the-art routing protocols in WSNs from classical routing protocols to swarm intelligence based protocols. The routing protocols are categorized based on their computational complexity, network structure, energy efficiency and path establishment. The second part of the paper presents a comparison of a representative number of classical and swarm based protocols. Comparing routing protocols in WSNs is currently a very challenging task for protocol designers. Often, much time is required to re-create and re-simulate algorithms from descriptions in published papers to perform the comparison. Compounding the difficulty is that some simulation parameters and performance metrics may not be mentioned. We see a need in the research community to have standard simulation and performance metrics for comparing different protocols. To this end, the final part of the paper resimulates different protocols using a Matlab based simulator: routing modeling application simulation environment (RMASE), and gives simulation results for standard simulation and performance metrics which we hope will serve as a benchmark for future comparisons for the research community. Hong, Sung-Hwa et al. [6] proposed the WSN (Wireless Sensor Network) algorithm which is applied sensor node that has low power consumption and efficiency measurement. Moreover, the efficiency routing protocol is proposed in this paper. The proposed algorithm reduces power consumption of sensor node data communication. It has not researched in LEACH (Low-Energy Adaptive Clustering Hierarchy) routing protocol. As controlling the active/sleep mode based on the measured data by sensor node, the energy consumption is able to be managed. In the event, the data is transferred to the local cluster head already set. We selected cluster head efficiently and uniformly distributed the energy to each cluster node through the proposed algorithm (EECS).Toumanari, Ahmed, and Rachid Latif [7] proposed a congestion-aware energy-saving routing protocol (CER) network. Specifically, a source node degree get obstruction of the surplus energy and neighbor node determine destination for packet. The experimental results show that there is a significant improvement in energy saving, network, and other energy output as-aware routing protocol. Yang, He et al. [8] improved the LEACH to an energy efficient multi-hop routing algorithm. In data transmission, it lowers energy consumption by inter-cluster and intra-cluster multi-hop transmission. The simulation runs the algorithm on NS2. The results show that the new algorithm's effectiveness in reducing energy consumption by comparing it with $\mathrm{LEACH}$, LEACH-C,DEEUC. Lizárraga, Evelia et al. [9] proposed an ant's partition method for (ACO), a meta-heuristic that is inspired in ant's behavior and how they collect their food. The proposed method equivalently divides the total number of ants in three different subsets and each one is evaluated separately by the corresponding variation of ACO (AS, EAS, MMAS) to solve different instances of The TSP. This method is based on the idea of "divide and conquer" to be applied in the division of the work, as the ants are evaluated in different ways in the same iteration. This method also includes a stagnation mechanism that stops at a certain variation if it's not working properly after several iterations. This allows us to save time performing tests and have less overhead in comparison with 
the conventional method, which uses just one variation of ACO in all iterations. Ünal, Muhammet et al. [10] discussed that main idea of ACO is to model the problem as the search for a minimum cost path in a graph. Artificial ants walk through this graph, looking for good paths. Each ant has a rather simple behavior so that it will typically only find rather poor-quality paths on its own. Better paths are found as the emergent result of the global cooperation among ants in the colony. Azharuddin, Md et al. [11] proposed distributed clustering and routing algorithms jointly referred as DFCR. The algorithm is shown to be energy efficient and fault tolerant. They performed extensive experiments on the proposed algorithm using various network scenarios. The experimental results are compared with the existing algorithms to demonstrate the strength of the algorithm in terms of various performance metrics. Ghaffari, Ali [12] proposed an new energy-efficient routing protocol (EERP) for WSNs using A-star algorithm. The proposed routing scheme improves the network lifetime by forwarding data packets via the optimal shortest path. Amgoth, Tarachand, and Prasanta K. Jana [13] proposed an energy aware routing algorithm for cluster based WSNs. The algorithm is based on a clever strategy of cluster head $(\mathrm{CH})$ selection, residual energy of the $\mathrm{CHs}$ and the intra-cluster distance for cluster formation. To facilitate data routing, a directed virtual backbone of $\mathrm{CHs}$ is constructed which is rooted at the sink. The proposed algorithm is also shown to balance energy consumption of the $\mathrm{CHs}$ during data routing process. We prove that the algorithm achieves constant message and linear time complexity. We test the proposed algorithm extensively. The experimental results show that the algorithm outperforms other existing algorithms in terms of network lifetime, energy consumption and other parameters. Kuila, Pratyay, and Prasanta K. Jana [14] presented Linear/Nonlinear Programming (LP/NLP) formulations of these problems followed by two proposed algorithms for the same based on particle swarm optimization (PSO). The clustering algorithm is presented by considering energy conservation of the nodes through load balancing. The proposed algorithms are experimented extensively and the results are compared with the existing algorithms to demonstrate their superiority in terms of network life, energy consumption, dead sensor nodes and delivery of total data packets to the base station. Helmy, Asmaa Osama et al. [15] proposed an optimized hierarchical routing technique which aims to reduce the energy consumption and prolong network lifetime. In this technique, the selection of optimal cluster heads (CHs) locations is based on Artificial Fish Swarm Algorithm (AFSA). Various behaviors in AFSA such as preying, swarming, and following are applied to select the best locations of CHs.. Simulation results show the stability and efficiency of the proposed technique. The results are obtained in terms of number of alive nodes and the energy residual mean value after some communication rounds. To prove the AFSA efficiency of energy consumption, we have compared it to LEACH and PSO. Simulation results show that the proposed method outperforms both LEACH and PSO in terms of first node die (FND) round, total data received by base station, network lifetime, and energy consume per round. Maryam, Sabet, and Naji HamidReza et al. [16] proposed a new decentralized hierarchical cluster-based routing algorithm for WSNs. The most of energy consumption occurs due to transmission of messages, such as data and control packets. In our new approach clustering and multi hop routing algorithms are performing at the same stage to decrease control packets. Simulation results show that the proposed protocol leads to reduction of sensor nodes' energy consumption and prolongs the network lifetime, significantly.

\section{PROPOSED METHODOLOGY}

Figure 2 shows the flowchart of the proposed methodology.

Step 1:- Initialize the wireless sensor network with the various characteristics.

Step 2:- Define sensor field with the respective placement of the sensor nodes and also the base station.

Step 3:- $\quad$ Now cluster head selection technique come in action to elect some of the sensor nodes as cluster heads.

Step 4:- $\quad$ Now association of the member nodes with the cluster heads will be done by using the minimum distance formula between the member nodes and the respective cluster heads.

Step 5:- $\quad$ Apply inter cluster data aggregation technique to fuse data from the cluster heads and compress it before sending to the base station.

Step 6:- $\quad$ Now evaluate the route using the Ant colony optimization and communicate the data from the cluster head(s) to the base station.

Step 7:- Count if any dead node and check whether all dead. If all dead the show network lifetime and return else move to step 3 




Fig 2: The flowchart of the proposed technique

\section{RESULTS AND DISCUSSIONS}

Result of ERA

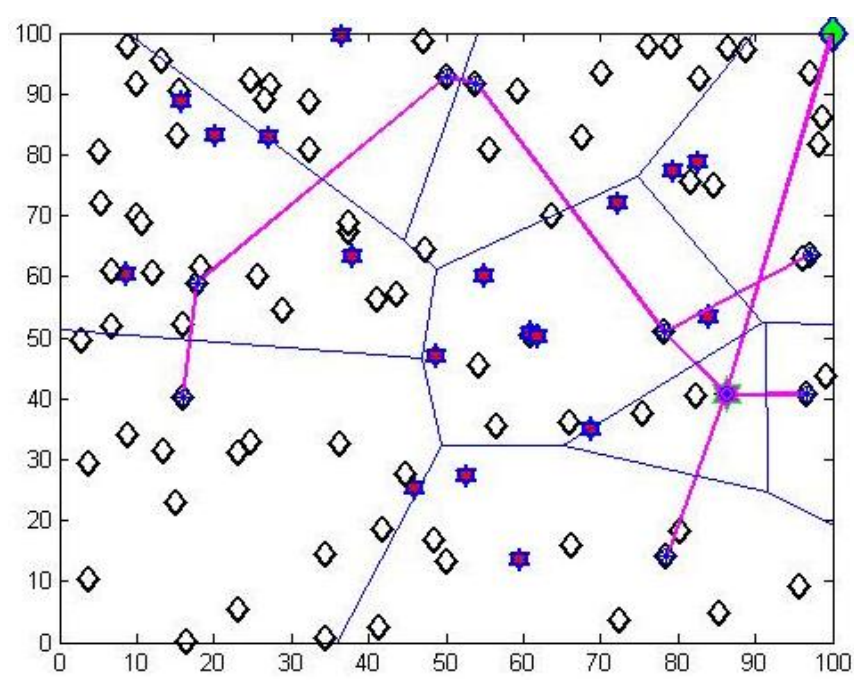

Fig 3: Network Model of ERA

Figure 3 is showing the area of sensor networks in which white diamond represents sensor nodes. Blue diamonds are cluster heads. Stared blue is depicting the cluster head and magenta lines are representing the communication between the cluster head and the base station and green diamond symbol is representing the base station and blue lines are depicting the cluster area.

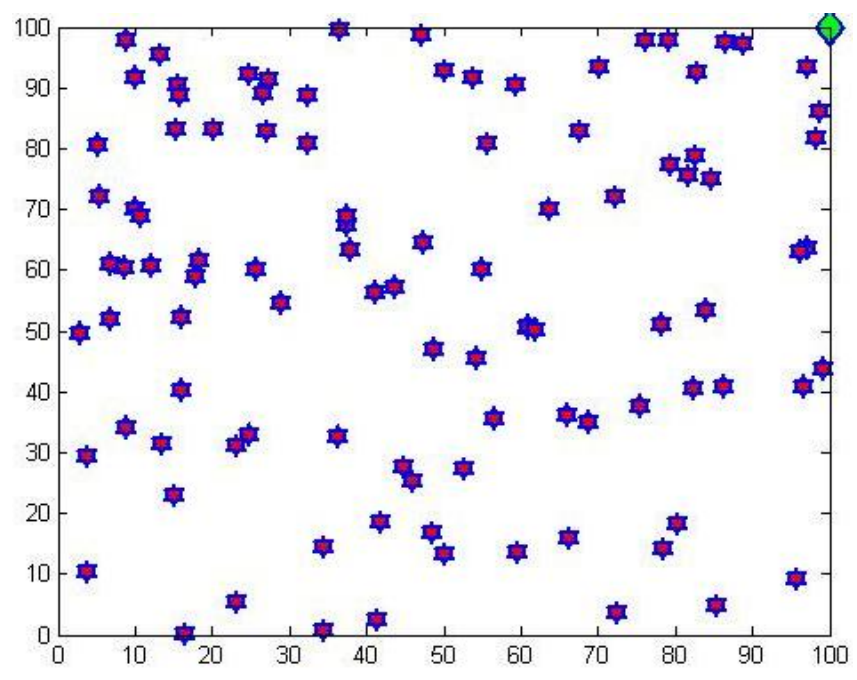

Fig 4: Dead nodes

Figure 4 is showing the dead network and at the end all of the nodes present in the network has become dead. The no. of dead nodes in the network is equal to total no. of nodes present in the network initially. In this case we have initially taken 100 nodes and total no. of dead nodes in the network at end are also 100 . 


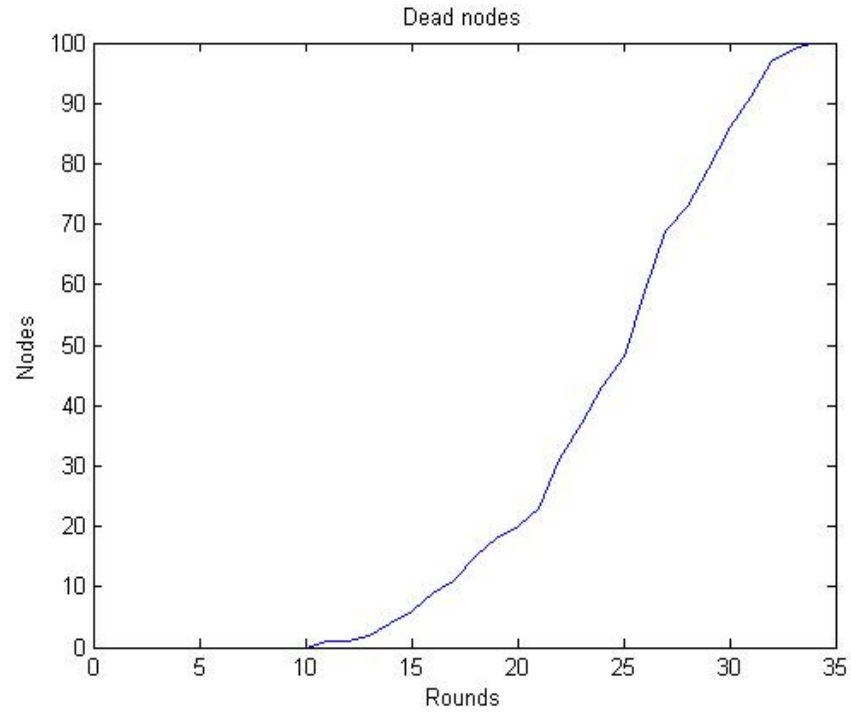

Fig 5: Graph of dead nodes

Figure 5 is clearly showing the dead nodes present in the network. It can be easily concluded from the graph that the time taken for first node to become dead is 10 rounds and last node has become dead at 34 .



Fig 6: Graph of remaining energy of nodes

Figure 6 is showing the remaining energy of the network. Initially the remaining energy of the network was full which was assumed to be 1 and as the no. of rounds goes on increasing the remaining energy goes on decreasing and after 34 rounds it has become zero.

\section{Result of ACOERA}

Figure 7 is showing the area of sensor networks in which white circle represents sensor nodes. Blue circles are cluster heads and blue lines are depicting the cluster area.

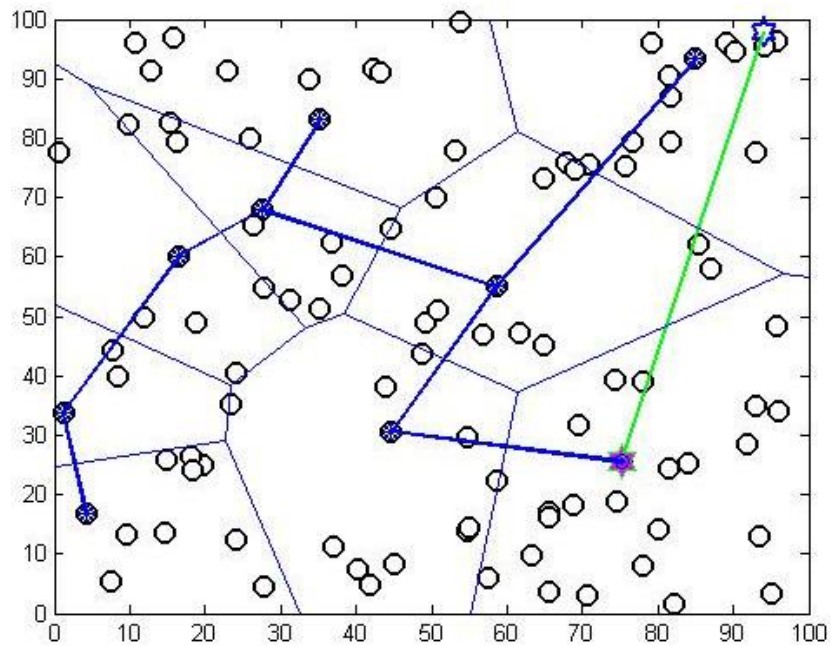

Fig 7: Network Model Of ACOERA

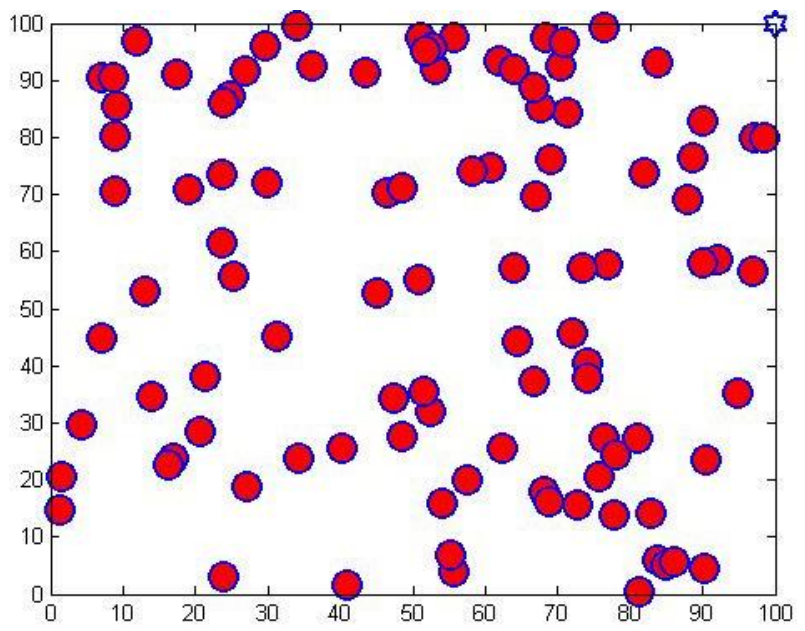

Fig 8: Dead nodes present in the network.

Figure 8 is depicting that all the nodes in the network has become dead. In this figure it is being shown that red circles are the dead nodes and in it no. of dead nodes are 100.



Fig 9: Graph of remaining energy of network.

Figure 9 is showing the remaining energy of the network. As no. of rounds goes on increasing the remaining energy of the network goes on decreasing and at the end remaining energy of the network has become zero. 


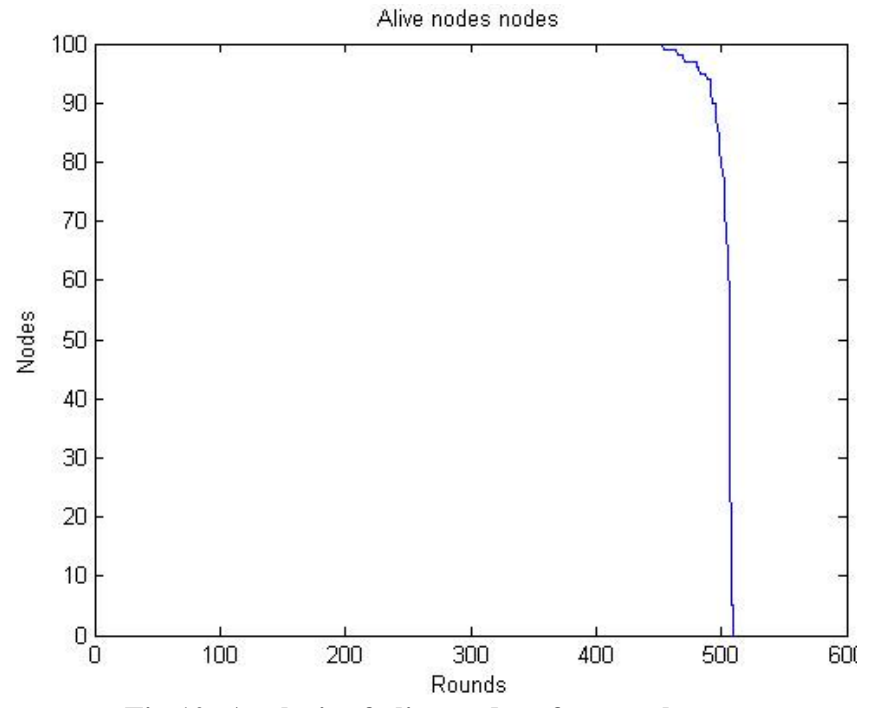

Fig 10: Analysis of alive nodes of network

Figure 10 is depicting the no. of alive nodes present in the network means the nodes which has not become dead. Initially all the nodes were alive till round 450 means at round 450 the no. of dead nodes were zero but as the rounds keeps on increasing the no. of alive nodes goes on decreasing and at the end of 500 rounds the no. of alive nodes in the network has become zero means now the total nodes present in the network has become dead.

\section{RESULTS OF Mobile ERA}

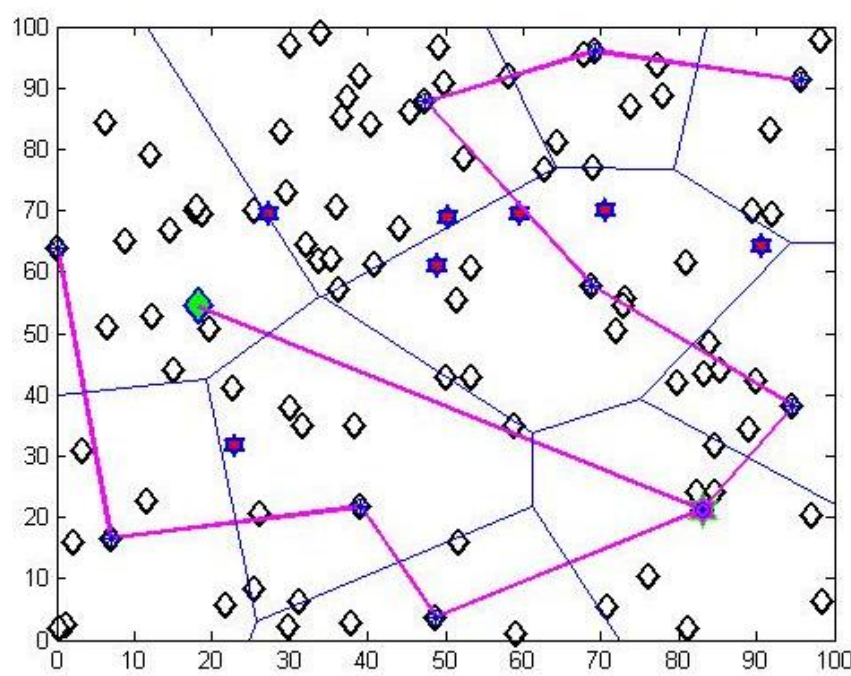

Fig 11: Network model of mobile ERA

Figure 11 is showing the area of sensor networks in which white diamond represents sensor nodes. Blue diamonds are cluster heads. Stared blue is depicting the cluster head and magenta lines are representing the communication between the cluster head and the base station and green diamond symbol is representing the base station and blue lines are depicting the cluster area.

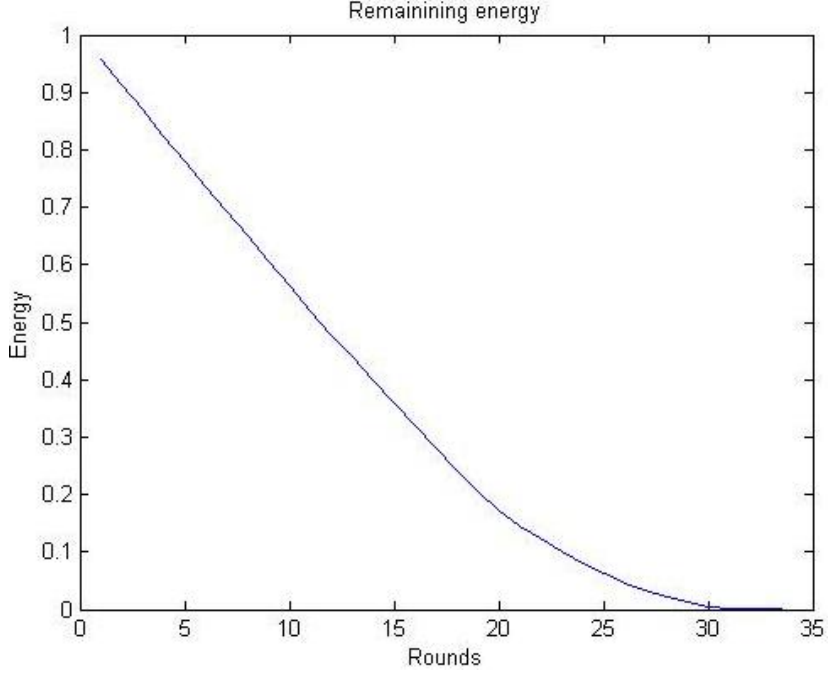

Fig 12: Analysis of remaining energy of nodes

Figure 12 is showing the remaining energy of the network of sensor nodes in which the remaining energy was assumed to be one initially at starting of zero round and as the no. of rounds goes on completing the remaining energy of the network goes on decreasing and at the end of $34^{\text {th }}$ round the total remaining energy of the network has become zero. And there is no more energy left in the network.

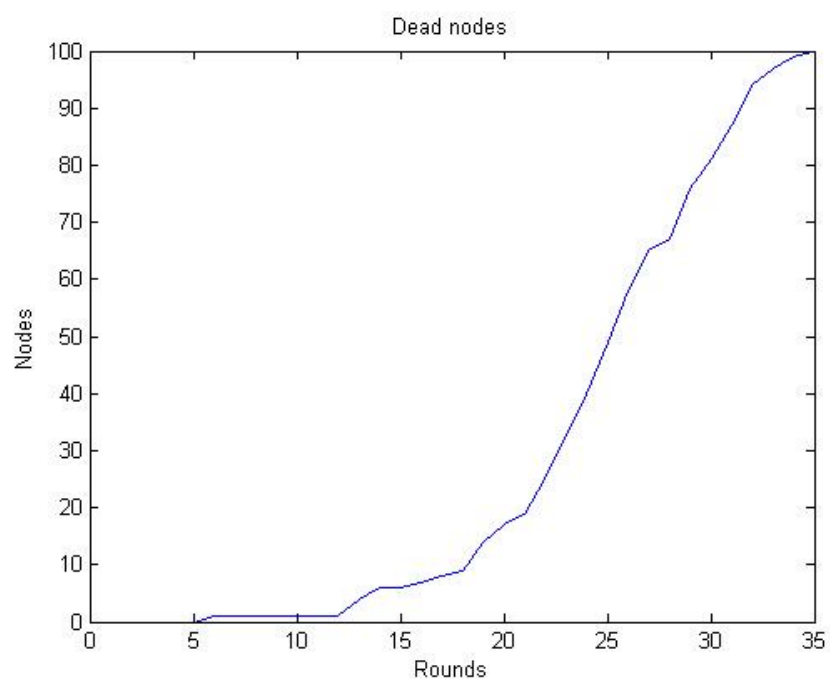

Fig 13: Analysis of dead nodes

Figure 13 is showing the no. of dead nodes present in the network .initially all the nodes are alive in the network means there is no dead nodes. At the end of $5^{\text {th }}$ round first node has become dead and at the end of $35^{\text {th }}$ round all the nodes present in the network has become dead, showing that at the end of $35^{\text {th }}$ round total no. of dead nodes are 100 . 




Fig 14: Alive nodes analysis

Figure 14 is showing the graph of alive nodes present in the network. Initially all the nodes present are alive and as the no. of rounds goes on increasing the no. of alive nodes goes on decreasing and hence no. of alive nodes becomes zero at the end.



Fig 15: Dead nodes analysis

Figure 15 is showing the no. of dead nodes. All the blue color diamonds are depicting the dead nodes and at the end whole of the nodes in the network are dead which is being shown in this figure and green diamond symbol is depicting the base station.

\section{RESULTS OF Mobile ACOERA}

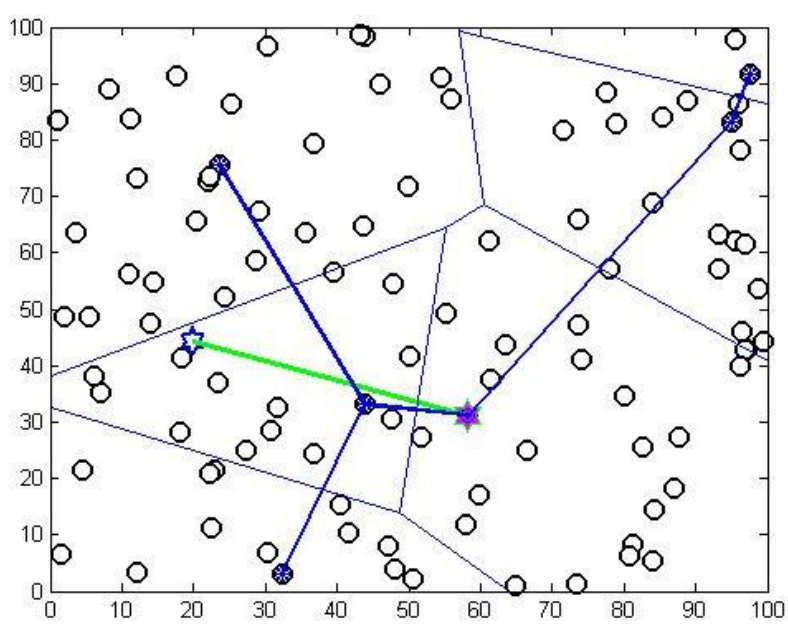

Fig 16: Mobile network of ACO ERA

Figure 16 is showing the area of sensor networks in which white circle represents sensor nodes. Blue diamonds are cluster heads. Stared blue is depicting the cluster head and blue lines are depicting the cluster area.

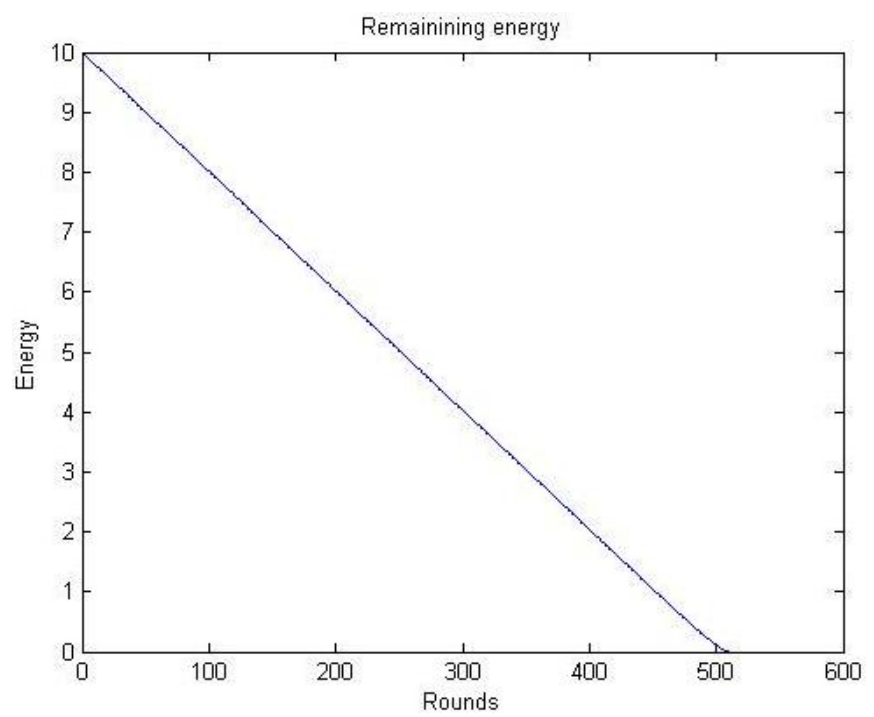

Fig 17: Analysis of remaining energy

Figure 17 is showing the remaining energy of the network. Initially energy was assumed to be full at the starting of the $0^{\text {th }}$ round and as the no. of rounds goes on increasing the remaining energy of the nodes goes on decreasing and at the end of the $500^{\text {th }}$ round the remaining energy of the network has become zero.

Figure 18 is showing the no. of alive nodes in the network at the no. of rounds increases the no. of alive nodes goes on decreasing and at the end no. of alive nodes present in the network are zero. 


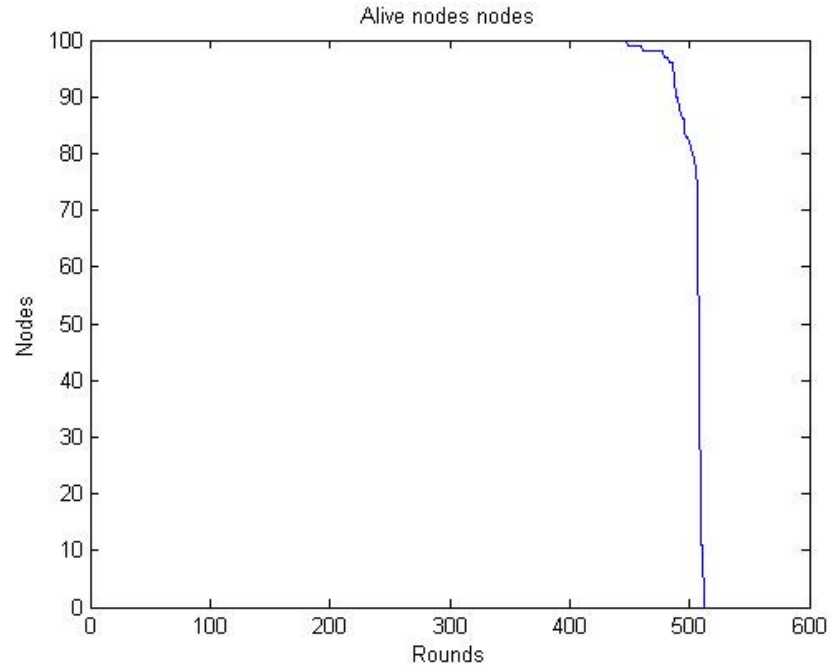

Fig 18: Alive node analysis

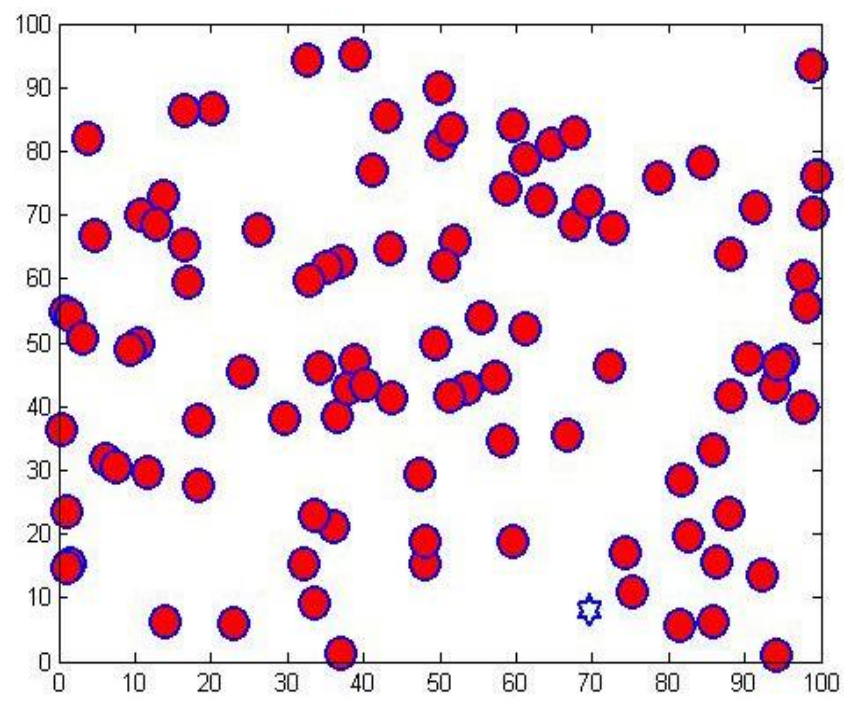

Fig 19: Dead nodes

Figure 19 is depicting that all the nodes in the network has become dead. In this figure it is being shown that red circles are the dead nodes and in it no. of dead nodes are 100

\section{Comparison}

Table 1 : Comparison Evaluation

\begin{tabular}{|l|l|l|l|l|}
\hline & ERA & ACOERA & $\begin{array}{l}\text { Mobile } \\
\text { ERA }\end{array}$ & $\begin{array}{l}\text { Mobile } \\
\text { ACOERA }\end{array}$ \\
\hline FND & 160 & 450 & 175 & 462 \\
\hline LND & 284 & 504 & 270 & 507 \\
\hline
\end{tabular}

FND ( FIRST NODE DEAD):- Table 2 shows the first node dead evaluation of the ERA, ACO_ERA, MOBILE ERA and MOBILE ACO_ERA protocols.
Table 2: Fast Node Dead Evaluation

\begin{tabular}{|l|l|l|l|l|}
\hline ENERGY & ERA & ACO_ERA & $\begin{array}{l}\text { MOBILE } \\
\text { ERA }\end{array}$ & $\begin{array}{l}\text { MOBILE } \\
\text { ACO_ERA }\end{array}$ \\
\hline 0.1 & 160 & 450 & 175 & 462 \\
\hline 0.11 & 195 & 521 & 159 & 519 \\
\hline 0.12 & 215 & 568 & 178 & 565 \\
\hline 0.13 & 233 & 614 & 200 & 612 \\
\hline 0.14 & 249 & 663 & 215 & 659 \\
\hline 0.15 & 263 & 711 & 244 & 708 \\
\hline 0.16 & 284 & 758 & 254 & 751 \\
\hline 0.17 & 306 & 806 & 276 & 797 \\
\hline 0.18 & 325 & 852 & 307 & 843 \\
\hline 0.19 & 350 & 899 & 324 & 890 \\
\hline 0.2 & 366 & 947 & 341 & 938 \\
\hline
\end{tabular}

LND (LAST NODE DEAD TIME):- Table 3 shows the last node dead evaluation of the ERA, ACO_ERA, MOBILE ERA and MOBILE ACO_ERA protocols.

Table 3: Last Node Dead Evaluation

\begin{tabular}{|c|c|c|c|c|}
\hline $\begin{array}{c}\text { ENERG } \\
\mathbf{Y}\end{array}$ & $\begin{array}{c}\text { ER } \\
\text { A }\end{array}$ & $\begin{array}{c}\text { ACO_ER } \\
\text { A }\end{array}$ & $\begin{array}{c}\text { MOBIL } \\
\text { E ERA }\end{array}$ & $\begin{array}{c}\text { MOBILE } \\
\text { ACO_ER } \\
\text { A }\end{array}$ \\
\hline 0.1 & 284 & 504 & 270 & 507 \\
\hline 0.11 & 317 & 557 & 297 & 572 \\
\hline 0.12 & 361 & 610 & 315 & 608 \\
\hline 0.13 & 356 & 654 & 353 & 662 \\
\hline 0.14 & 399 & 711 & 374 & 710 \\
\hline 0.15 & 473 & 753 & 393 & 779 \\
\hline 0.16 & 475 & 802 & 426 & 816 \\
\hline 0.17 & 483 & 861 & 445 & 866 \\
\hline 0.18 & 555 & 916 & 480 & 918 \\
\hline 0.19 & 565 & 955 & 495 & 975 \\
\hline 0.2 & 590 & 0 & 531 & 0 \\
\hline
\end{tabular}




\section{CONCLUSION AND FUTURE SCOPE}

In this paper, the review has shown that the most of the existing technique has neglected the issues like the effects of the mobile sink in the most of the energy efficient protocols has been ignored. Moreover the effect of lossless data compression has been neglected by the most of the researchers. Also no optimization technique is considered for the effective route selection in ERA protocol. Therefore to overcome these issues, an Ant colony optimization based energy efficient routing algorithm (AERA) has been proposed Also the performance of AERA and ERA under mobile sink based wireless sensor networks has been evaluated. The comparison has been drawn between ERA, and proposed AERA based on the following parameters like First node dead time, Network life time, Dead nodes, Remaining energy, Packets sent to base station and Packets sent to cluster head.

\section{REFERENCES}

[1] Li, Li, Shu-song Dong, and Xiang-ming Wen. "An energy efficient clustering routing algorithm for wireless sensor networks." The journal of China Universities of posts and Telecommunications 13, no. 3 (2006): 71-75.

[2] Jin, Yan, Ju-Yeon Jo, Ling Wang, Yoohwan Kim, and Xiaozong Yang. "ECCRA: An energy-efficient coverage and connectivity preserving routing algorithm under border effects in wireless sensor networks." Computer Communications31, no. 10 (2008): 2398-2407.

[3] Jang, Yong-Jae, Si-Yeong Bae, and Sung-Keun Lee. "An energy-efficient routing algorithm in wireless sensor networks." In Future Generation Information Technology, pp. 183-189. Springer Berlin Heidelberg, 2011.

[4] Zeydan, Engin, Didem Kivanc, Cristina Comaniciu, and Ufuk Tureli. "Energy-efficient routing for correlated data in wireless sensor networks." Ad Hoc Networks 10, no. 6 (2012): 962-975.

[5] Zungeru, Adamu Murtala, Li-Minn Ang, and Kah Phooi Seng. "Classical and swarm intelligence based routing protocols for wireless sensor networks: A survey and comparison." Journal of Network and Computer Applications 35, no. 5 (2012): 1508-1536.

[6] Hong, Sung-Hwa, Byoung-Kug Kim, and Joon-Min Gil. "An Algorithm for an Energy-Efficient Smart Sensor with EECS Routing Protocol in Wireless Sensor Networks." In Embedded and Multimedia Computing Technology and Service, pp. 685-692. Springer Netherlands, 2012.
[7] Toumanari, Ahmed, and Rachid Latif. "Energy Efficient Routing Algorithm in Wireless Sensor Networks." Computer Engineering and Intelligent Systems 4, no. 5 (2013): 60-69.

[8] Yang, He, Jia Xu, Ruchuan Wang, and Liyang Qian. "Energy-Efficient Multi-hop Routing Algorithm Based on LEACH." In Advances in Wireless Sensor Networks, pp. 578-587. Springer Berlin Heidelberg, 2013.

[9] Lizárraga, Evelia, Oscar Castillo, and José Soria. "A method to solve the traveling salesman problem using ant colony optimization variants with ant set partitioning." In Recent Advances on Hybrid Intelligent Systems, pp. 237-246. Springer Berlin Heidelberg, 2013.

[10] Ünal, Muhammet, Ayça Ak, Vedat Topuz, and Hasan Erdal. "Ant Colony Optimization (ACO)." In Optimization of PID Controllers Using Ant Colony and Genetic Algorithms, pp. 31-35. Springer Berlin Heidelberg, 2013.

[11] Azharuddin, Md, Pratyay Kuila, and Prasanta K. Jana. "Energy efficient fault tolerant clustering and routing algorithms for wireless sensor networks."Computers \& Electrical Engineering (2014).

[12] Ghaffari, Ali. "An Energy Efficient Routing Protocol for Wireless Sensor Networks using A-star Algorithm." Journal of Applied Research and Technology12, no. 4 (2014): 815-822.

[13] Amgoth, Tarachand, and Prasanta K. Jana. "Energyaware routing algorithm for wireless sensor networks." Computers \& Electrical Engineering (2014).

[14] Kuila, Pratyay, and Prasanta K. Jana. "Energy efficient clustering and routing algorithms for wireless sensor networks: Particle swarm optimization approach."Engineering Applications of Artificial Intelligence 33 (2014): 127-140.

[15] Helmy, Asmaa Osama, Shaimaa Ahmed, and Aboul Ella Hassenian. "Artificial Fish Swarm Algorithm for EnergyEfficient Routing Technique." In Intelligent Systems' 2014, pp. 509-519. Springer International Publishing, 2015.

[16] Maryam, Sabet, and Naji HamidReza. "A Decentralized Energy Efficient Hierarchical Cluster-based Routing Algorithm for Wireless Sensor Networks."AEUInternational Journal of Electronics and Communications (2015). 